\title{
Implante de Stent Não-Valvado na Via de Saída do Ventrículo Direito: Forma Simples e Efetiva de Retardar Nova Intervenção Cirúrgica
}

\author{
Raul Ivo Rossi Filho', João Luiz L. Manica', Mônica S. Borges ${ }^{1}$, Paulo Renato M. Machado'
}

\section{RESUMO}

Introdução: A ampliação da via de saída do ventrículo direito (VSVD), com ou sem condutos, é frequente no tratamento de doenças cardíacas congênitas. Entretanto, a durabilidade desses condutos é restrita e o implante de stent se torna uma boa opção para desobstrução da VSVD. O objetivo deste estudo é relatar a experiência de um centro terciário no implante de stent não-valvado e seus resultados a curto e médio prazos. Método: Entre setembro de 2000 e janeiro de 2009, 11 pacientes com indicação de reintervenção cirúrgica receberam stents para desobstrução da VSVD. A média de idade no momento do procedimento foi de $12,5 \pm 8,3$ anos (1-28 anos) e o peso médio foi de $35 \pm 20,9 \mathrm{~kg}(6-62 \mathrm{~kg})$. A média de seguimento do estudo foi de $12,5 \pm 6$ meses (0-48 meses). Resultados: A média da pressão sistólica no ventrículo direito diminuiu de $99 \pm 23 \mathrm{mmHg}$ (pré-procedimento) para $57 \pm 12 \mathrm{mmHg}$ após o implante do stent $(p<0,001)$, assim como, ao ecocardiograma transtorácico, o gradiente ventrículo direito-artéria pulmonar (VD-AP) caiu de $69 \pm$ $19 \mathrm{mmHg}$ para $33 \pm 20 \mathrm{mmHg}(\mathrm{p}<0,01)$. Em $40 \%$ dos casos houve necessidade de reintervenção, em média, 22 meses após o procedimento. Houve um caso de fratura de stent, um caso de insucesso e um óbito não relacionado ao procedimento. Conclusão: A angioplastia da VSVD com stent não-valvado é uma medida paliativa, altamente efetiva e segura, capaz de retardar a necessidade de reintervenção cirúrgica. Além disso, permite o crescimento somático dos pacientes jovens e a redução do dano ao ventrículo direito para futuro implante de stents valvados para tratamento da insuficiência pulmonar livre.

DESCRITORES: Contenedores. Cardiopatias congênitas. Implante de prótese vascular.

\begin{abstract}
Non-Valved Stent Implantation in Right Ventricle Outflow Tract: a Simple Way to Postpone New Surgical Intervention

Background: Right ventricle outflow tract (RVOT) reconstruction is frequently used to repair cardiac malformations. However, the lifetime of these conduits is short and stent implantation is a good option to relieve obstructed RVOT. The objective of this study is to report the experience of a tertiary center with non-valved stent implantation and its short and medium term results. Methods: Between September 1990 and January 2009, 11 late postoperative patients were submitted to stent implantation due to RVOT obstruction. Mean age at the time of procedure was $12.5 \pm$ 8.3 years ( $1-28$ years), and mean weight was $35 \pm 20.9 \mathrm{~kg}$ $(6-62 \mathrm{~kg})$. Mean follow-up time was $12.5 \pm 6$ months $(0-48$ months). Results: Mean systolic right ventricular pressures decreased from $99 \pm 23 \mathrm{mmHg}$ before to $57 \pm 12 \mathrm{mmHg}$ ( $p<0.001)$ after stent implantation. At echocardiogram, $\mathrm{RV}$ to PA gradient decreased from $69 \pm 19 \mathrm{mmHg}$ to $33 \pm$ $20 \mathrm{mmHg}(\mathrm{p}<0.01)$. Forty percent of the patients required percutaneous or surgical reintervention during a mean time of 22 months after the procedure. There was one case of stent fracture, relieved with the implantation of another stent, one case of failure, and one death not related to the procedure. Conclusion: Non-valved stent implantation in obstructed RVOT is a highly effective and safe palliative approach. Moreover, it allows the somatic growth of young patients and does not preclude future percutaneous implantation of valved stents to treat free pulmonary regurgitation.
\end{abstract}

DESCRIPTORS: Stents. Heart defects, congenital. Blood vessel prosthesis implantation.

\footnotetext{
1 Serviço de Cardiologia Intervencionista em Cardiopatias Congênitas do Instituto de Cardiologia do Rio Grande do Sul/Fundação Universitária de Cardiologia - Porto Alegre, RS, Brasil.

Correspondência: Raul Ivo Rossi Filho. Av. Princesa Isabel, 370 Santana - Porto Alegre, RS, Brasil - CEP 90620-001

E-mail: rossi.pesquisa@cardiologia.org.br

Recebido em: 3/1/2009 • Aceito em: 24/3/2009
} 
A ampliação da via de saída do ventrículo direito (VSVD), com ou sem implante de condutos entre o ventrículo direito (VD) e a artéria pulmonar (AP), tem se tornado muito frequente no tratamento de diversas doenças cardíacas congênitas em diferentes faixas etárias. Entretanto, a durabilidade dessas ampliações, principalmente em lactentes jovens, é muito restrita em decorrência da desproporção progressiva causada pelo crescimento somático, bem como da redução eventual do calibre do conduto com subsequente sobrecarga pressórica do lado direito do coração $0^{1-3}$. A necessidade de múltiplas intervenções cirúrgicas é a regra, o que acarreta aumento da morbidade e da dificuldade técnica dos procedimentos futuros ${ }^{2}$.

Ao contrário da baixa efetividade da angioplastia percutânea com balão para desobstrução de condutos entre o $\mathrm{VD}$ e a $A \mathrm{P}^{4-6}$, o implante de stent não-valvado tem demonstrado bons resultados como tratamento paliativo, associando-se a queda imediata do gradiente VD-AP e diminuição da sobrecarga pressórica do $\mathrm{VD}^{7,8}$. O advento de stents valvados ${ }^{9-12}$ permitiu a desobstrução da VSVD, além de proteger o VD da insuficiência pulmonar total consequente ao implante dos stents comuns ${ }^{13}$. Essa nova opção, que será introduzida brevemente em nosso meio, tem suas próprias dificuldades, tais como a necessidade de implante prévio de conduto ou tubo de até $22 \mathrm{~mm}$, o calibre ainda grande dos sistemas de entrega e, finalmente, seu elevado custo $^{10}$. Essa situação torna o uso de stents não-valvados uma excelente opção para o retardo temporal de novas intervenções cirúrgicas em todas as faixas etárias.

O presente estudo descreve a experiência de um serviço terciário no implante de stents não-valvados em pacientes com obstrução na VSVD de diferentes etiologias e descreve os resultados obtidos na tentativa de prolongar a vida útil de condutos previamente implantados.

\section{MÉTODO}

\section{Amostra}

A amostra compreende uma série de casos retrospectiva de todos os pacientes submetidos a angioplastia com implante de stent na VSVD no Serviço de Cardiologia Intervencionista em Cardiopatias Congênitas do Instituto de Cardiologia do Rio Grande do Sul/Fundação Universitária de Cardiologia (Porto Alegre, RS). Entre setembro de 2000 e janeiro de 2009, 11 pacientes com obstrução da VSVD com indicação de intervenção foram encaminhados para implante de stent com o objetivo de retardar nova intervenção cirúrgica. A média de idade no momento do procedimento foi de $12,5 \pm 8,3$ anos (1-28 anos) e o peso médio foi de 35 $\pm 20,9 \mathrm{~kg}(6-62 \mathrm{~kg})$.

Pressão sistólica estimada no VD (PSVD) acima de dois terços da pressão sistêmica e sinais de sobrecarga volumétrica do VD estimados por ecocardiografia transtorácica ou gradiente pico-a-pico maior que $40 \mathrm{mmHg}$ no laboratório de hemodinâmica, independentemente da presença de sintomas, foram considerados indicações para o procedimento. A presença de insuficiência pulmonar foi avaliada inicialmente por ecocardiografia transtorácica; entretanto, nos casos com implante de conduto e má janela ecocardiográfica, o grau de regurgitação foi avaliado na sala de hemodinâmica.

\section{Procedimento e técnica}

Inicialmente, realiza-se criterioso estudo manométrico e angiográfico nas projeções ântero-posterior e perfil esquerdo, com injeções de contraste no VD e na VSVD para adequada visualização da obstrução com cateter angiográfico Multi-Track ${ }^{\mathrm{TM}}$ (NuMED Inc. Hopkinton, Estados Unidos). Os dados hemodinâmicos, como PSVD, gradiente pico-a-pico VD-AP e razão entre a PSVD e a pressão sistêmica, foram medidos antes e após o implante do stent. Não foi realizada punção arterial de rotina e a pressão sistêmica foi habitualmente avaliada por meio do manguito. O grau de calcificação do conduto e a localização principal da lesão foram avaliados pelo estudo angiográfico, bem como os diâmetros do conduto antes e após o procedimento.

A técnica para o implante de stent em condutos foi descrita previamente na literatura ${ }^{14,15}$. Consiste na introdução de stent manualmente montado em balão, através de bainha longa Mullins (Cook Medical Inc. Bloomington, Estados Unidos) sobre guia rígida adequadamente posicionada distalmente na árvore pulmonar. O diâmetro do balão escolhido (12-20 mm) corresponde a aproximadamente $110 \%$ do maior diâmetro medido no estudo angiográfico. A projeção perfil esquerdo $\left(\mathrm{OAE} 90^{\circ}\right)$ é a mais comumente utilizada para tomada de decisão em relação ao diâmetro do balão e ao comprimento do stent. O comprimento do stent é decidido com o objetivo de cobrir a lesão estenótica com adequado apoio distal e proximal na VSVD e tronco da AP. Múltiplas injeções através da bainha permitem adequado posicionamento do stent para sua liberação. A liberação do stent é realizada por meio de insuflação manual com eventual pós-dilatação até 12 atms de maneira controlada. Após o implante, cateter Multi-Track ${ }^{\mathrm{TM}}$ é inserido para verificação de gradiente residual e aspecto angiográfico final (Figura 1). A pré-dilatação com balão não é realizada de rotina, podendo ser empregada nos casos de lesões graves calcificadas. Além disso, coronariografia seletiva para avaliação da proximidade do stent com as artérias coronárias também não é realizada rotineiramente, exceto nos casos com trajeto anômalo de artéria coronária através da VSVD ou alteração eletrocardiográfica durante a pré-dilatação.

Consentimento informado foi obtido antes de todos os procedimentos. 

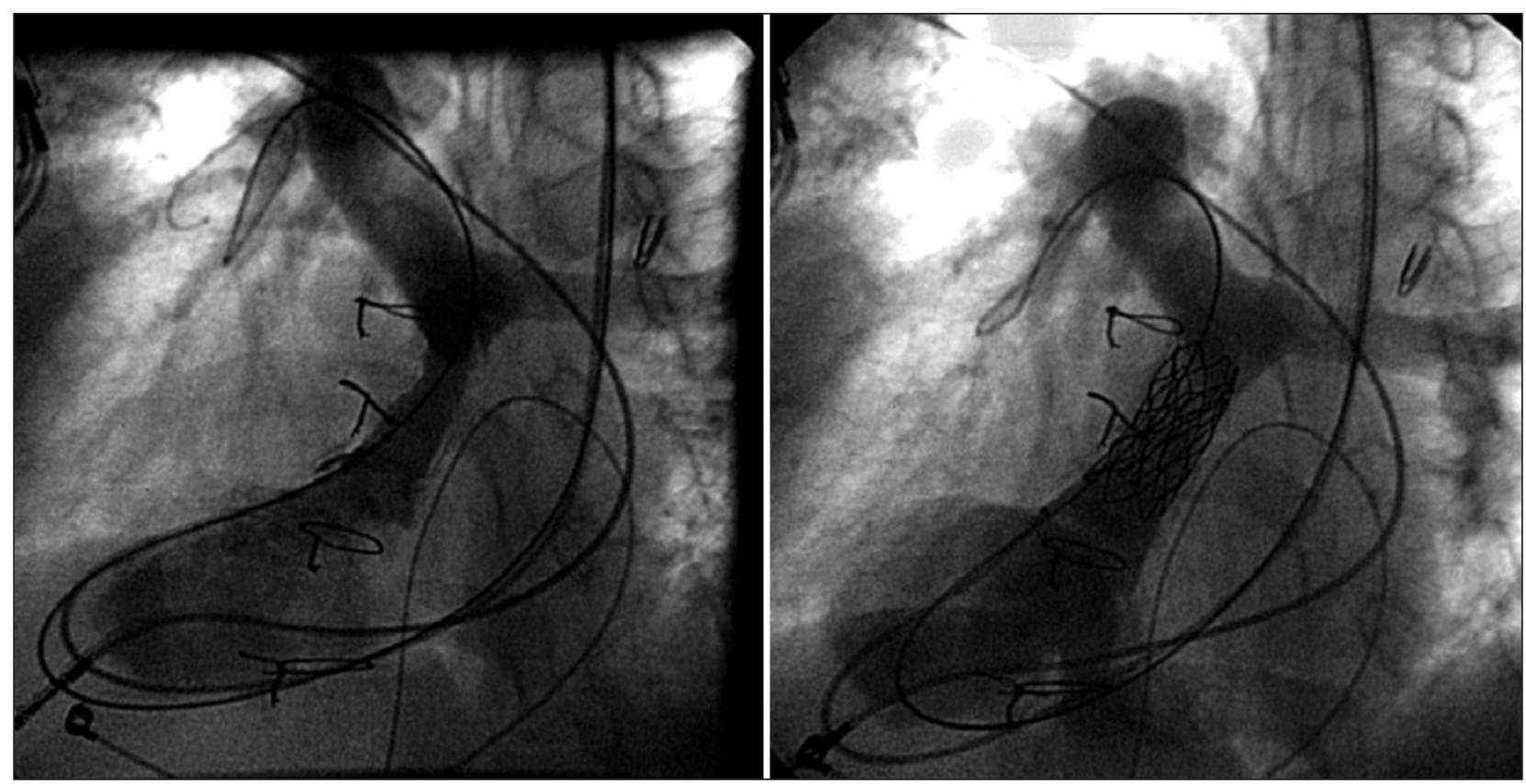

Figura 1 - Resultado imediato de stent implantado em paciente portadora de dextrocardia, isomerismo esquerdo, dupla via de saída do ventrículo direito com aorta anterior e estenose pulmonar, pós-operatório tardio de cirurgia de Rastelli com homoenxerto na via de saída do ventrículo direito e marca-passo.

\section{Seguimento}

Os pacientes foram seguidos no ambulatório de cardiologia pediátrica da instituição e os dados clínicos e ecocardiográficos foram comparados com os dados obtidos previamente ao implante do stent. Novo cateterismo era realizado se a ecocardiografia sugerisse presença de reestenose significativa (PSVD estimada maior que $60 \%$ da pressão sistêmica).

\section{Análise estatística}

Dados clínicos e hemodinâmicos foram descritos, como frequência, média e desvio padrão. Para análise das variáveis contínuas foi utilizado o teste $t$ de Student para amostras pareadas. Valores de $p<0,05$ foram considerados estatisticamente significativos. A análise estatística foi realizada com o uso do Statistical Package for Social Sciences versão 11.0.

\section{RESULTADOS}

\section{Pacientes}

Os dados demográficos, descritos a seguir, estão apresentados, de forma resumida, na Tabela 1: 5 pacientes eram portadores de tetralogia de Fallot ou atresia pulmonar com comunicação interventricular (2 corrigidos com retalho transanular e 3 submetidos a implante de homoenxerto); 2 (um com implante de homoenxerto e outro com tubo de dácron) tinham diagnóstico de dupla VSVD (DVSVD); 2 apresentavam transposição de grandes vasos (ambos submetidos a cirurgia de
Jatene); e 2, anomalia de situs e DVSVD (um com homoenxerto e outro com tubo de dácron). Calcificação acentuada dos condutos foi identificada em $66 \%$ dos homoenxertos (Figura 2) e em 50\% dos tubos de dácron (um caso), não sendo evidenciada nos pacientes submetidos a retalho transanular e operação de Jatene. Todos os pacientes apresentavam, ao ecocardiograma transtorácico ou à angiografia, insuficiência pulmonar ao menos moderada.

A lesão era predominantemente supravalvar (91\% dos casos) e o diâmetro médio dos balões utilizados $(15,2 \mathrm{~mm})$ foi muito semelhante à média do maior diâmetro da VSVD medida na incidência de perfil $(14,1 \mathrm{~mm})$. Em 4 (36\%) pacientes foram realizados, em procedimento prévio, dilatação com balão sem stent, porém sem adequada desobstrução, persistindo a indicação de intervenção. Em todos os casos foi realizado implante direto do stent, e 8 pacientes apresentavam sintomas de dispneia ou intolerância aos esforços previamente ao procedimento.

\section{Dados hemodinâmicos e angiográficos}

Durante o cateterismo, observou-se queda imediata da PSVD, em média, de $40 \pm 9 \%$ em relação à pressão aferida previamente ao implante do stent (99 vs. $57 \mathrm{mmHg}$; $\mathrm{p}<0,001)$. Quando avaliada a razão entre a PSVD e a pressão sistêmica, observou-se queda estatisticamente significativa após o implante do stent (92\% antes vs. 55\% após o procedimento; $p<0,001)$ (Figura 3). Angiograficamente, a medida do menor diâmetro obtido na incidência ântero-posterior aumentou 
TABELA 1

Dados demográficos

\begin{tabular}{|c|c|c|c|c|c|c|c|c|c|}
\hline Paciente & Sexo & $\begin{array}{l}\text { Diagnóstico } \\
\text { (idade, anos) }\end{array}$ & Cirurgia & $\begin{array}{c}\text { Calcificação } \\
\text { difusa }\end{array}$ & $\begin{array}{l}\text { Tempo até } \\
\text { implante } \\
\text { do stent }\end{array}$ & $\begin{array}{l}\text { Idade no } \\
\text { cat (anos) }\end{array}$ & Stent & Sucesso & $\begin{array}{c}\text { Reintervenção } \\
\text { (meses) }\end{array}$ \\
\hline 1 & $\mathrm{~F}$ & DVSVD & $\begin{array}{c}\text { BAP (5 meses), } \\
\text { homoenxerto } \\
\text { (12 anos) }\end{array}$ & $\mathrm{N}$ & 10,2 anos & 22,2 & $\mathrm{CP} 45 \mathrm{~mm}$ & S & $\begin{array}{c}\mathrm{S} \\
\text { Homoenxerto } \\
(2 \text { meses })\end{array}$ \\
\hline 2 & $M$ & $\mathrm{~T} 4 \mathrm{~F}$ & $\begin{array}{c}\text { Reparo T4F } \\
\text { (1,1 ano) }\end{array}$ & $\mathrm{N}$ & 2 dias & 1,1 & $\begin{array}{c}\text { Express } \\
\text { LD } 17 \mathrm{~mm}\end{array}$ & S & $\begin{array}{c}\text { Óbito } \\
\text { (causa não- } \\
\text { cardíaca) }\end{array}$ \\
\hline 3 & $\mathrm{~F}$ & TGV, CIV & $\begin{array}{l}\text { Operação } \\
\text { de Jatene } \\
\text { (3 meses) }\end{array}$ & $\mathrm{N}$ & 1,9 ano & 2,2 & CP $28 \mathrm{~mm}$ & $S$ & $\mathrm{~N}$ \\
\hline 4 & $M$ & DVSVD, EP & $\begin{array}{l}\text { Tubo de } \\
\text { dácron } \\
(2,7 \text { anos })\end{array}$ & $\mathrm{N}$ & 5,1 anos & 7,8 & $\begin{array}{c}\text { Palmaz } \\
308\end{array}$ & S & $\begin{array}{c}\mathrm{S} \\
\text { Homoenxerto } \\
\text { (41 meses) }\end{array}$ \\
\hline 5 & $M$ & $\mathrm{AP}, \mathrm{CIV}$ & $\begin{array}{c}\text { Reparo patch } \\
\text { autólogo } \\
(1,5 \text { ano), } \\
\text { reoperação patch } \\
\text { (5,5 anos) }\end{array}$ & 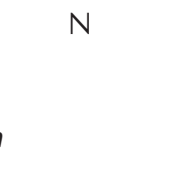 & 5,2 anos & 10,7 & $\begin{array}{l}\text { ITI MAX } \\
\text { LD } 36 \text { mm }\end{array}$ & S & $\begin{array}{c}\text { S } \\
\text { Angioplastia } \\
\text { com balão } \\
\text { (25 meses) }\end{array}$ \\
\hline 6 & $M$ & $\mathrm{~T} 4 \mathrm{~F}$ & $\begin{array}{c}\text { Reparo T4F } \\
\text { (1,8 ano), } \\
\text { homoenxerto } \\
(2,7 \text { anos })\end{array}$ & $S$ & 2,2 anos & 4,9 & $\begin{array}{c}\text { Dynamic } \\
25 \mathrm{~mm}\end{array}$ & $S$ & $\begin{array}{c}\text { S } \\
\text { Angioplastia } \\
\text { com stent } \\
\text { (13 meses) }\end{array}$ \\
\hline 7 & F I & $\begin{array}{c}\text { Isom E, DVSVD, } \\
\text { Ao ant, EP }\end{array}$ & $\begin{array}{c}\text {, Homoenxerto } \\
\text { (10,5 anos) }\end{array}$ & S & 5,5 anos & 16 & CP 34 mm & $S$ & $\mathrm{~N}$ \\
\hline 8 & $M$ & TGV, CIV & $\begin{array}{l}\text { Operação } \\
\text { de Jatene } \\
\text { (3 meses) }\end{array}$ & $\mathrm{N}$ & 13 anos & 13,3 & CP $28 \mathrm{~mm}$ & S & $\mathrm{N}$ \\
\hline 9 & $\mathrm{~F}$ & SI, DVSVD, EP & $\begin{array}{l}\text { Tubo de } \\
\text { dácron } \\
\text { (6,4 anos), } \\
\text { tubo de } \\
\text { dácron } \\
\text { (19,2 anos) }\end{array}$ & $S$ & 9 anos & 28 & CP 22 mm & $\begin{array}{l}\mathrm{N} \\
\text { (implantado } \\
\text { na veia } \\
\text { femoral } \\
\text { comum) }\end{array}$ & - \\
\hline 10 & $\mathrm{~F}$ & AP, CIV & $\begin{array}{l}\text { Homoenxerto } \\
\qquad(5 \text { anos })\end{array}$ & S & 8 anos & 13 & CP 34 mm & S & $\mathrm{N}$ \\
\hline 11 & $\mathrm{~F}$ & $\mathrm{AP}, \mathrm{CIV}$ & $\begin{array}{c}\text { Shunt BT } \\
\text { (18 dias), } \\
\text { homoenxerto } \\
\text { (2 anos), } \\
\text { homoenxerto } \\
\text { (8,1 anos) }\end{array}$ & S & 5,3 anos & 13,4 & $\mathrm{CP} 45 \mathrm{~mm}$ & S & $\mathrm{N}$ \\
\hline
\end{tabular}

Ao ant = aorta anterior; $\mathrm{AP}=$ atresia pulmonar; $\mathrm{BAP}=$ bandagem da artéria pulmonar; $\mathrm{BT}=\mathrm{Blalock}-\mathrm{Taussig}$; cat $=$ cateterismo; $\mathrm{CIV}=$ comunicação interventricular; DVSVD = dupla via de saída do ventrículo direito; $E P=$ estenose pulmonar; $F=$ feminino; Isom $E$ = isomerismo esquerdo; $\mathrm{M}=$ masculino; $\mathrm{N}=$ não; $\mathrm{S}=\mathrm{sim} ; \mathrm{SI}=$ situs inversus; T4F = tetralogia de Fallot; TGV = transposição dos grandes vasos.

significativamente após o implante do stent, passando de $12 \pm 2,6 \mathrm{~mm}$ para $17 \pm 7,5 \mathrm{~mm}(\mathrm{p}<0,05)$.

Ao compararmos os pacientes submetidos a reintervenção percutânea ou cirúrgica durante o seguimento do estudo, observamos tendência a maiores médias de PSVD obtidas logo após o implante do stent nos pacientes que necessitaram de reintervenção, comparativamente aos pacientes que não necessitaram de reintervenção $(70 \pm 12 \mathrm{mmHg}$ vs. $50 \pm 9 \mathrm{mmHg}$, respectivamente; $\mathrm{p}=0,06$ ) (Figura 4 ). 


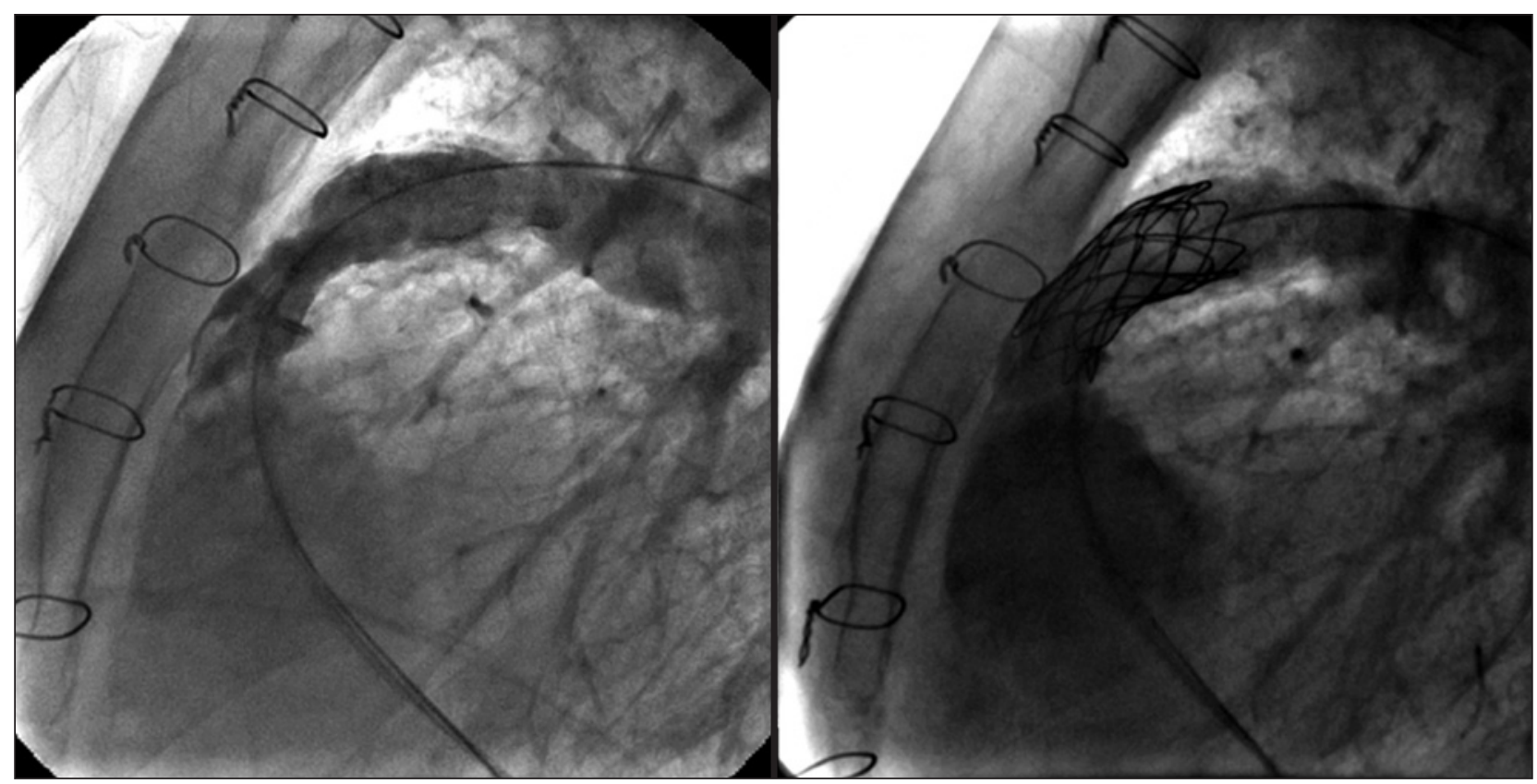

Figura 2 - Stent implantado em paciente portadora de atresia pulmonar com comunicação interventricular com homoenxerto difusamente calcificado e excelente resultado imediato.

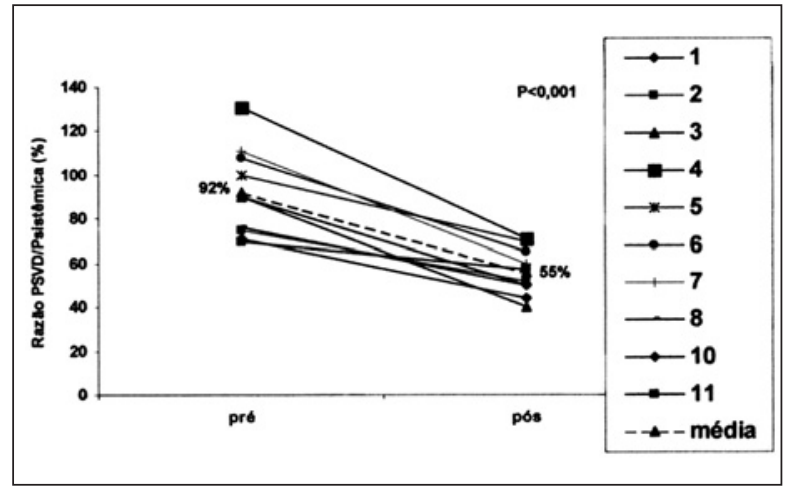

Figura 3 - Razão entre a pressão sistólica no ventrículo direito e a pressão sistêmica (PSVD/Psistêmica) medidas no laboratório de hemodinâmica antes e imediatamente após o implante do stent.

\section{Complicações e insucessos}

Um paciente portador de tetralogia de Fallot (paciente 6), previamente corrigido com retalho transanular e, posteriormente, com homoenxerto, evoluiu com sinais de sobrecarga pressórica do VD, adequadamente aliviada após implante de stent na VSVD. Entretanto, após um ano, evidências de reestenose aos exames clínico e ecocardiográfico justificavam a realização de novo cateterismo. Ao exame, demonstrou-se fratura do stent e PSVD de $99 \mathrm{mmHg}$ (suprassistêmica). Foi implantado novo stent Palmaz Gênesis $19 \mathrm{~mm}$ montado em balão $12 \mathrm{~mm}$, com excelente resultado imediato e queda da PSVD para $50 \%$ da pressão sistêmica.

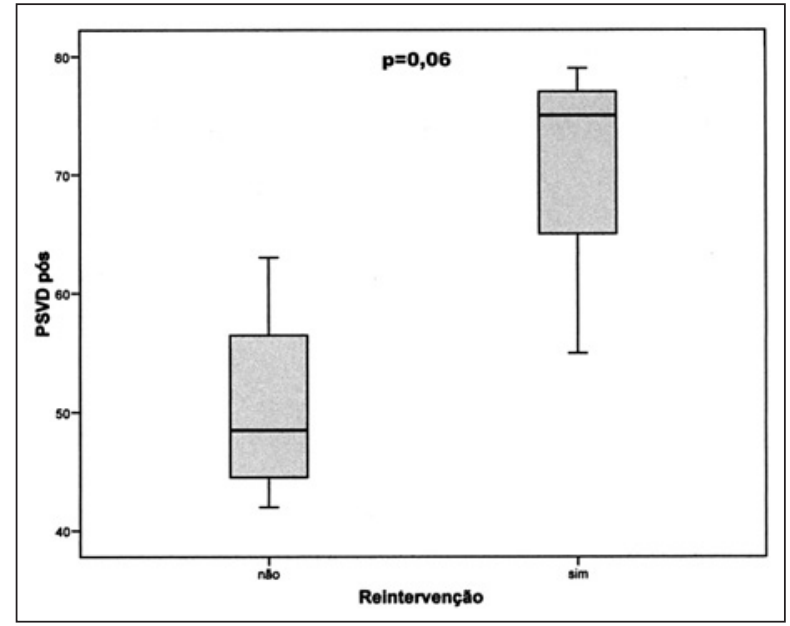

Figura 4 - Pressão sistólica no ventrículo direito (PSVD) medida no laboratório de hemodinâmica imediatamente após o implante do stent nos pacientes que necessitaram de reintervenção, comparativamente aos pacientes que não necessitaram de reintervenção.

Em uma paciente portadora de situs inversus e DVSVD com estenose pulmonar (paciente 9), submetida a duas operações prévias para implante de conduto na VSVD, não foi possível a liberação do stent no local adequado. O dispositivo não alcançou a lesão, e, por estar parcialmente expandido, foi liberado na veia femoral comum, sem maiores complicações.

O único óbito ocorreu por causa não relacionada ao procedimento. O paciente, no pós-operatório imediato de retalho transanular para tetralogia de Fallot 
(paciente 2), em insuficiência renal aguda e acidose refratária, recebeu stent na VSVD dois dias após a cirurgia, porém não resistiu às comorbidades e faleceu dez dias depois.

\section{Seguimento}

A média de seguimento do estudo foi de 12,5 \pm 6 meses (0-48 meses). Entre os 8 pacientes sintomáticos em que o stent foi implantado com sucesso, 6 melhoraram, 1 permaneceu sintomático e 1 não teve seguimento suficiente para tal avaliação.

Houve queda significativa do gradiente instantâneo máximo estimado pelo pico de velocidade sistólica na VSVD ao ecocardiograma transtorácico realizado previamente ao procedimento, em relação ao exame ecocardiográfico de controle (média de $69 \pm 19 \mathrm{mmHg}$ vs. $33 \pm 20 \mathrm{mmHg}$, respectivamente; $\mathrm{p}<0,01$ ) (Figura 5).

Em 40\% dos pacientes houve necessidade de reintervenção percutânea ou cirúrgica, em média 20 meses após angioplastia percutânea com stent. Em 2 pacientes portadores de DVSVD submetidos a cirurgia de Rastelli e implante de stent para desobstrução tardia da VSVD (119 e 61 meses após, respectivamente) foi necessária a substituição do conduto após 2 e 48 meses do procedimento percutâneo, respectivamente (em um deles após tentativa de redilatação com balão). Não houve dificuldade técnica para a abordagem cirúrgica em decorrência da presença dos stents na VSVD. Outros 2 pacientes, um com atresia pulmonar com comunicação interventricular e outro com tetralogia de Fallot, com implante de stent 63 e 27 meses após o procedimento cirúrgico, respectivamente, também necessitaram reintervenção. $O$ primeiro necessitou de dilatação percutânea do stent 25 meses após e atualmente não tem indicação de reintervenção. No segun-

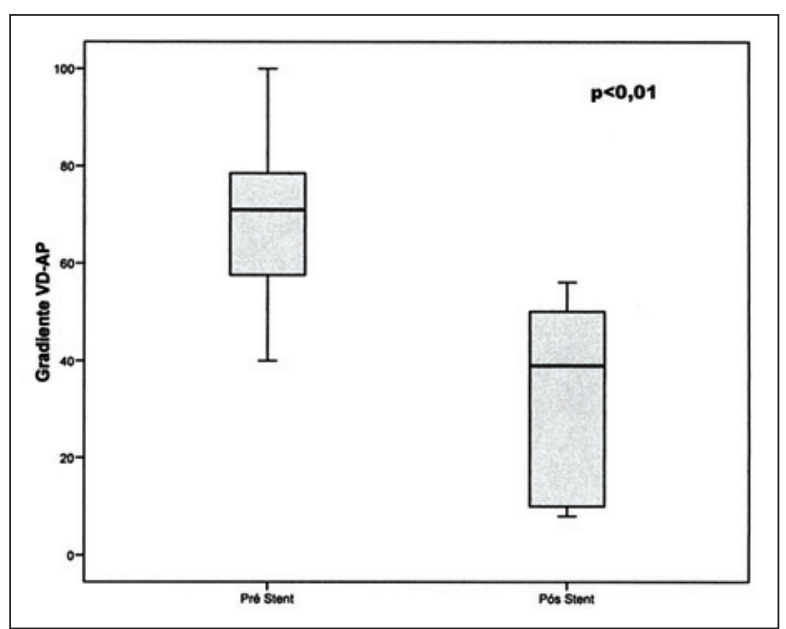

Figura 5 - Gradiente ventrículo direito-artéria pulmonar (VD-AP) estimado por ecocardiografia transtorácica antes e após o implante do stent na via de saída do ventrículo direito. do caso, já descrito previamente, o stent fraturou-se e foi implantado novo stent com sucesso.

A média de seguimento dos pacientes que não necessitaram de reintervenção foi de 7,2 \pm 3,2 meses.

\section{DISCUSSÃO}

Estudos prévios demonstram a sobrevida restrita de condutos na VSVD, principalmente em lactentes jovens e pacientes previamente submetidos a outros procedimentos cirúrgicos ${ }^{1}$. As múltiplas intervenções cirúrgicas a que são submetidos esses pacientes, na maioria dos casos por obstrução progressiva, e a boa tolerabilidade do VD à sobrecarga volumétrica causada pela insuficiência pulmonar ${ }^{8,16}$ motivaram a busca por alternativas capazes de retardar a sobrevida desses condutos ${ }^{17}$. A angioplastia com balão não se mostrou efetiva $^{4-6}$ e o implante de stent tem demonstrado ser, a curto e médio prazos, uma boa opção capaz de desobstruir a VSVD, diminuindo a PSVD e retardando a necessidade de reintervenção cirúrgica sem risco de piora da função ventricular direita ${ }^{7}$.

Em nosso estudo, angioplastia com stent foi a terapêutica de escolha em pacientes com diagnósticos distintos submetidos a diversos tipos de ampliação da VSVD. Esse fato demonstra a versatilidade dessa alternativa paliativa, independentemente da presença ou não de condutos previamente implantados.

O fato de apenas $40 \%$ dos pacientes serem submetidos, em procedimento prévio, a dilatação com balão é justificado pela baixa efetividade dessa alternativa terapêutica com altos índices de reestenose precoce descritos na literatura ${ }^{4-6}$.

A diferença entre as médias do menor diâmetro dos condutos medidos antes $(12 \mathrm{~mm})$ e após a dilatação com stent $(17 \mathrm{~mm})$, ainda que estatisticamente significativa $(p<0,05)$, está possivelmente subestimada pelo fato de a medida ter sido realizada na incidência ântero-posterior. De acordo com Sugiyama et al. ${ }^{16}$, o menor diâmetro do conduto é demonstrado na projeção lateral em decorrência da compressão anterior do esterno e posterior da aorta ascendente. Isso explica a discrepância entre o valor médio do diâmetro do balão utilizado para a dilatação com stent (15,2 mm), obtido a partir da projeção de perfil, e o valor médio do menor diâmetro ao final do procedimento, a partir da projeção ântero-posterior $(17 \mathrm{~mm})$. Não foi possível a comparação dos dados com a projeção de perfil em decorrência da falta de dados.

Todos os pacientes apresentavam, ao ecocardiograma transtorácico ou à angiografia, regurgitação pulmonar ao menos moderada. A queda imediata da PSVD, evidenciada durante o cateterismo após o implante do stent, é um dado extremamente importante, principalmente em um espectro de pacientes com esse grau de insuficiência pulmonar. Estudos prévios de- 
monstram que a sobrecarga volumétrica produzida pela insuficiência pulmonar total, previamente presente ou provocada pelo stent, é bem tolerada nos pacientes com obstrução residual mínima ${ }^{8,18}$. Além disso, nenhum paciente apresentava lesões residuais na árvore pulmonar periférica, o que poderia agravar a repercussão hemodinâmica da insuficiência pulmonar grave $^{19}$.

Entre os pacientes que necessitaram reintervenção após o implante do stent, a média da PSVD ao final do procedimento foi maior que a média nos pacientes que não tiveram necessidade de reintervenção no seguimento do estudo $(70 \pm 12 \mathrm{mmHg}$ vs. $50 \pm 9 \mathrm{mmHg}$, respectivamente; $p=0,06)$. Apesar de estatisticamente não-significativo, esse achado vai ao encontro dos dados da literatura, que demonstram que a PSVD imediatamente após o implante do stent é um fator de risco para reintervenção ${ }^{7,8}$.

Vários outros fatores capazes de predizer o risco de reintervenção cirúrgica após o implante de stent foram descritos na literatura, como idade precoce no momento do stent, presença de homoenxertos e condutos com menos de $10 \mathrm{~mm}$ de diâmetro ${ }^{7,8}$. Por causa do tamanho da amostra, não foi possível estabelecer fatores de risco capazes de predizer a necessidade de nova reintervenção cirúrgica nesse estudo.

Entretanto, o fato de os dois pacientes que necessitaram reintervenção cirúrgica serem portadores de condutos (homoenxerto e tubo de dácron) corrobora os estudos prévios que demonstram que esses implantes estão sujeitos a maior calcificação difusa, o que pode ser um fator de risco para necessidade de reintervenção cirúrgica mais precoce por obstrução progressiva $^{14,18}$. Não houve relato de dificuldade técnica durante a reintervenção cirúrgica pela presença do stent implantado na VSVD, assim como não há relatos na literatura que sustentem essa hipótese. No presente estudo, todos os pacientes com calcificação acentuada na VSVD eram portadores de condutos, não sendo evidenciado nos pacientes submetidos a correção com retalho autólogo ou switch arterial. Além disso, stents implantados em condutos entre o VD e a AP estão mais predispostos a fraturas, possivelmente, pela maior deposição de cálcio e pela compressão do esterno sobre o conduto ${ }^{14,18}$. Em nossa casuística, tivemos um diagnóstico de fratura em paciente com implante prévio de homoenxerto aórtico em posição pulmonar, que se encontrava difusamente calcificado, determinando significativa obstrução da VSVD. Essa lesão foi adequadamente controlada após implante de novo stent sobre o local da fratura. Dados da literatura referentes à efetividade da reintervenção percutânea após implante de stent são controversos ${ }^{7,8,18}$, porém a presença de fatores específicos, como compressão ânteroposterior do esterno e utilização de balões com diâmetro inadequado no primeiro procedimento, identifica os pacientes suscetíveis a nova dilatação ${ }^{16}$. Em nosso estudo, dois dos três casos submetidos a nova intervenção percutânea, com ou sem implante de novo stent, permanecem livres de reintervenção cirúrgica: em um paciente previamente corrigido com retalho autólogo e lesão localizada e em outro paciente previamente descrito com fratura do stent. Esses dados reforçam a idéia da efetividade da reintervenção percutânea em casos individualizados que se beneficiam dessa alternativa com retardo da indicação cirúrgica.

Raras complicações são descritas na literatura, dentre as quais as mais temidas são embolia e malposição do stent em VD ou árvore pulmonar, podendo resultar em necessidade de remoção cirúrgica da prótese ${ }^{7,8}$. No presente estudo, em um paciente não foi possível adequado posicionamento do stent sob a lesão, o qual, por estar parcialmente expandido, foi liberado na veia femoral comum sem maiores intercorrências. O paciente que faleceu dez dias após o implante do stent se encontrava no segundo dia de pós-operatório de tetralogia de Fallot com insuficiência renal aguda e acidose refratária, sendo submetido a implante de stent na VSVD com sucesso. A pressão sistólica imediatamente após o implante, medida no laboratório de hemodinâmica, caiu de $80 \mathrm{mmHg}$ para $55 \mathrm{mmHg}$ e o ecocardiograma realizado cinco dias após o procedimento mostrava gradiente VD-AP de $32 \mathrm{mmHg}$. Apesar do sucesso terapêutico do procedimento, o paciente não resistiu às intercorrências clínicas.

Coronariografia seletiva para avaliação da proximidade da artéria coronária com o conduto é realizada principalmente nos casos em que o conduto foi implantado em pacientes com trajeto anômalo da artéria descendente anterior através da VSVD ${ }^{8}$. Entretanto, alguns serviços realizam essa investigação rotineiramente $^{7}$. Neste estudo, coronariografia não foi realizada em nenhum paciente, pois não houve alteração eletrocardiográfica durante a realização da pré-dilatação, além de não haver descrição prévia de trajeto anômalo de artéria coronária. Não foi evidenciada nenhuma intercorrência isquêmica durante o procedimento.

Mesmo com tempo de seguimento restrito, o fato de que todos os pacientes tinham indicação cirúrgica no momento da intervenção percutânea comprova a efetividade do procedimento em diferentes defeitos cardíacos congênitos submetidos a ampliação da VSVD, com ou sem implante de condutos externos. Neste estudo, foram observadas maior suscetibilidade à calcificação difusa e maior incidência de fraturas de stents nos homoenxertos e tubos externos, apesar do pequeno número de pacientes, corroborando os dados da literatura. Entretanto, esse fato não diminui a importância da angioplastia com stent nesse grupo específico de pacientes, permanecendo a indicação em diversas situações. Além disso, a VSVD desobstruída pelo implante de stent não-valvado permite o crescimento somático dos pacientes jovens e a redução do 
dano no VD para um futuro implante de stents valvados ou novo conduto valvado, no intuito de tratar a insuficiência pulmonar total $9,12,13$. Outro benefício obtido pela presença de stent na via de saída, no algoritmo de tratamento com stents valvados, é a maior rigidez e o maior suporte conferidos à via de saída, necessários para o implante de stent com valva ${ }^{10,11}$.

Apesar do pequeno número de pacientes, podemos concluir que a angioplastia da VSVD com stent não-valvado é uma medida paliativa altamente efetiva e segura, capaz de retardar a necessidade de reintervenção cirúrgica.

\section{CONFLITO DE INTERESSES}

Os autores declararam inexistência de conflito de interesses.

\section{REFERÊNCIAS BIBLIOGRÁFICAS}

1. Stark J, Bull C, Stajevic M, Jothi M, Elliott M, de Leval M. Fate of subpulmonary homograft conduits: determinants of late homograft failure. J Thorac Cardiovasc Surg. 1998;115(3): 506-16.

2. Mohammadi S, Belli E, Martinovic I, Houyel L, Capderou A, Petit J, et al. Surgery for right ventricle to pulmonary artery conduit obstruction: risk factors for further reoperation. Eur J Cardiothorac Surg. 2005;28(2):217-22.

3. Baskett RJ, Ross DB, Nanton MA, Murphy DA. Factors in the early failure of cryopreserved homograft pulmonary valves in children: preserved immunogenicity? J Thorac Cardiovasc Surg. 1996;112(5):1170-9.

4. Zeevi B, Keane JF, Perry SB, Lock JE. Balloon dilation of postoperative right ventricular outflow obstructions. J Am Coll Cardiol. 1989;14(2):401-12.

5. Lloyd TR, Marvin WJ Jr., Mahoney LT, Lauer RM. Balloon dilation valvuloplasty of bioprosthetic valves in extracardiac conduits. Am Heart J. 1987;114(2):268-74.

6. Sohn S, Kashani IA, Rothman A. Partial and transient relief of conduit obstruction by low-pressure balloon dilation in patients with congenital heart disease. Cathet Cardiovasc Diagn. 1995;34(1):35-40,

7. Peng LF, McElhinney DB, Nugent AW, Powell AJ, Marshall AC, Bacha EA, et al. Endovascular stenting of obstructed right ventricle-to-pulmonary artery conduits: a 15-year experience. Circulation. 2006;113(22):2598-605.

8. Ovaert C, Caldarone CA, McCrindle BW, Nykanen D, Freedom RM, Coles JG, et al. Endovascular stent implantation for the management of postoperative right ventricular outflow tract obstruction: clinical efficacy. J Thorac Cardiovasc Surg. 1999;118(5):886-93.

9. Bonhoeffer P, Boudjemline $Y$, Qureshi SA, Le Bidois J, Iserin $\mathrm{L}$, Acar $\mathrm{P}$, et al. Percutaneous insertion of the pulmonary valve. J Am Coll Cardiol. 2002;39(10):1664-9.

10. Khambadkone S, Bonhoeffer P. Nonsurgical pulmonary valve replacement: why, when, and how? Catheter Cardiovasc Interv. 2004;62(3):401-8.

11. Khambadkone S, Coats L, Taylor A, Boudjemline Y, Derrick $G$, Tsang $V$, et al. Percutaneous pulmonary valve implantation in humans: results in 59 consecutive patients. Circulation. 2005;112(8):1189-97.

12. Lurz P, Coats L, Khambadkone S, Nordmeyer J, Boudjemline $Y$, Schievano $S$, et al. Percutaneous pulmonary valve implantation: impact of evolving technology and learning curve on clinical outcome. Circulation. 2008;117(15):1964-72.

13. Coats L, Khambadkone S, Derrick G, Sridharan S, Schievano S, Mist B, et al. Physiological and clinical consequences of relief of right ventricular outflow tract obstruction late after repair of congenital heart defects. Circulation. 2006;113(17):2037-44.

14. Powell AJ, Lock JE, Keane JF, Perry SB. Prolongation of RVPA conduit life span by percutaneous stent implantation. Intermediate-term results. Circulation. 1995;92(11):3282-8.

15. Sabedotti M, Huber J, Guimarães NMO, Rossi Filho RI. Stents na circulação pulmonar. Rev Bras Cardiol Invas. 2005; 13(3):146-52.

16. Sugiyama H, Williams W, Benson LN. Implantation of endovascular stents for the obstructive right ventricular outflow tract. Heart. 2005;91(8):1058-63.

17. Hosking MC, Benson LN, Nakanishi T, Burrows PE, Williams WG, Freedom RM. Intravascular stent prosthesis for right ventricular outflow obstruction. J Am Coll Cardiol. 1992; 20(2):373-80.

18. Pedra CA, Justino $H$, Nykanen DG, VanArsdell G, Coles JG, Williams WG, et al. Percutaneous stent implantation to stenotic bioprosthetic valves in the pulmonary position. J Thorac Cardiovasc Surg. 2002;124(1):82-7.

19. Ilbawi MN, Idriss FS, DeLeon SY, Muster AJ, Gidding SS, Berry TE, et al. Factors that exaggerate the deleterious effects of pulmonary insufficiency on the right ventricle after tetralogy repair. Surgical implications. J Thorac Cardiovasc Surg. 1987;93(1):36-44. 